Editorial

\title{
Relapsing Polychondritis and Large-vessel Vasculitis
}

\author{
David P. D’Cruz ${ }^{1}\left({ }^{0}\right.$ and Marcela A. Ferrada ${ }^{2}$
}

Relapsing polychondritis (RP) is a rare and in some cases fatal systemic inflammatory rheumatic disorder characterized by episodic inflammation of cartilage ${ }^{1,2,3}$. Common clinical features include chondritis of the nasal bridge, auricular cartilage, ocular and inner ear inflammation, arthritis, and involvement of the tracheobronchial tree. Destruction of the laryngeal and tracheal cartilage rings may lead to collapse of the airways and is associated with a high risk of morbidity and mortality ${ }^{4}$. Its rarity often leads to considerable delay in establishing a diagnosis ${ }^{1,2,3}$.

RP may present with similar clinical features to other autoimmune rheumatic diseases such as granulomatosis with polyangiitis (GPA) and eosinophilic GPA (eGPA). Treatment for RP is usually with corticosteroids and immunosuppressive drugs but there are no randomized trials or specific guidelines for management, so treatment remains empirical and based on expert opinion $^{5}$.

Vascular involvement in RP ranges from 5\% to $25 \%$. The disease can affect small, medium, and large vessels. Although aortic involvement is particularly rare, it is associated with significant morbidity and mortality. The largest study in the literature evaluating aortic involvement by Le Besnerais, et al of 172 patients with RP found a prevalence of nonatheromatous aortic disease in 11 patients $(6.4 \%)^{6}$. The pattern of disease included isolated aortitis, aortic aneurysms, aortic ectasia, and aortic dissection. The thoracic aorta was most commonly affected, followed by the abdominal aorta. Cardiac and extraaortic vascular involvement was more common in RP patients with aortic disease. Aortic lesions most often develop later in the disease course, although it is possible that aortic disease may be missed at presentation because it is often asymptomatic.

The study by Tomelleri and colleagues in this issue of The

'D.P. D'Cruz, MD, FRCP, Louise Coote Lupus Unit, Guy's Hospital, London, UK; ${ }^{2}$ M.A. Ferrada, MD, US National Institutes of Health, Bethesda, Maryland, USA.

Address correspondence to Prof. D. D'Cruz, Consultant Rheumatologist, Louise Coote Lupus Unit, Guy's Hospital, London SE1 9RT, UK. Email:david.d'cruz@kcl.ac.uk.

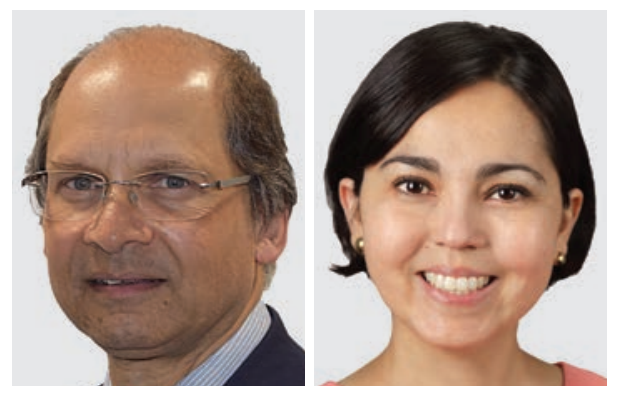

Journal highlights the importance of aortic disease in $\mathrm{RP}^{7}$. In their cohort of 41 patients, 4 (9.7\%) had large-vessel vasculitis (LVV) affecting the aorta and its branches. Their literature review found a further 17 patients with RP, and LVV most often developed later in the disease course. Disease involving the subclavian, carotid, iliac, renal, vertebral, axillary, femoral, inferior mesenteric arteries, and the brachiocephalic trunk occurred. Imaging modalities included conventional angiography in the majority of the patients as well as computed tomography (CT), magnetic resonance (MR) angiography, and positron emission tomography (PET)/CT. An important observation of their study is that most of the patients were female (62\%) and young (median $28 \mathrm{yrs}$, range 7-59), perhaps indicating that this particular population is at a higher risk of developing vascular involvement. Recognition of large-vessel involvement in patients with RP may help prevent further complications, considering that large-vessel involvement is associated with significant morbidity and an increased risk of premature mortality ${ }^{5,6}$.

These studies raise important discussion points. Should clinicians routinely screen for LVV at diagnosis and at intervals during the disease course of RP? The increasing use of PET/CT may facilitate early diagnosis both of RP and LVV Fluorodeoxyglucose (FDG) uptake indicating cartilage inflammation and vascular inflammation can be seen readily on PET scanning, and the corresponding CT images may delineate aortitis, aneurysms, and ectasia as well as document the extent and distribution of large-vessel disease? ${ }^{9}$. An obvious caveat, however, is that corticosteroid use may suppress FDG uptake.

Many patients with newly diagnosed RP, especially where airway disease is suspected, undergo dynamic CT scanning of the respiratory tract to assess airway inflammation as well as tracheal collapse. These scans may also detect aortic disease and could be a useful screening tool for LVV at diagnosis.

The ideal methods of screening for LVV in patients with RP are not clear and who should be screened is also debatable. The studies cited here suggest that patients with cardiac involvement are more likely to have large-vessel vascular disease, so

\section{See Vasculitis in RP, page 1780}


perhaps the focus should be on those patients. The tools used for screening should minimize or avoid radiation exposure, so MR angiography of the aorta and its main branches could be useful. Color duplex ultrasound scanning of the aorta and the peripheral vasculature is similarly noninvasive and could be undertaken annually. There are, however, no consensus guideline recommendations on the necessity, timing, and duration of followup imaging for patients with evidence of LVV in RP or indeed in diseases such as giant cell arteritis or Takayasu arteritis (TA), although the European League Against Rheumatism guidelines are useful ${ }^{5,10}$.

The management of non-atheromatous large-vessel disease in $\mathrm{RP}$ depends on the presenting features, the extent of the vascular disease, and associated cardiac involvement, and is of necessity multidisciplinary. As with LVV, medical management with conventional therapies such as corticosteroids and immunosuppressive agents are the first-line therapies. Patients requiring surgical intervention for aortic disease such as aneurysms, dissection, and aortic root dilatation resulting in aortic regurgitation should be managed intensively with medical therapies to ensure good disease control prior to surgery. Even so, the complication rates following vascular surgery in patients with RP are significant. In the study by Le Besnerais, et al, aortic complications were responsible for death in up to $27 \%$ of patients with $\mathrm{RP}^{6}$.

The current accepted nomenclature system for vasculitis resulted from the 2012 Chapel Hill Consensus Conference $(\mathrm{CHCC})^{11}$. The nomenclature included new terms and categories of variable vessel vasculitis and secondary forms of vasculitis such as Behçet disease and Cogan syndrome; these 2 conditions share several similarities with RP and they can overlap. For example, patients with MAGIC (mouth and genital ulcers with inflammation of cartilage) syndrome interestingly have a higher risk of developing aortitis, posing the question of the importance of recognizing particular clinical features as risk factors for LVV.

Presently, RP is not included in the CHCC vasculitis nomenclature. There is arguably a strong case to include RP in the current vasculitis nomenclature for many reasons. These include the prevalence of LVV in RP as highlighted by these recent studies, as well as several reports demonstrating that the etiology of some of the clinical manifestations in RP could be vasculitis. The associations between vasculitic diseases such as TA, polyarteritis nodosa, GPA, IgA-associated vasculitis, and eGPA with polychondritis are also relevant.
$\mathrm{RP}$ is an exceptionally rare inflammatory rheumatic disease, which makes study of the disease, its management, and prognosis difficult. The increasing appreciation that LVV may occur in these patients should prompt strategies to detect cardiovascular involvement at presentation and during the disease course to reduce the risk of vascular damage and improve outcomes.

\section{REFERENCES}

1. Horváth A, Páll N, Molnár K, Kováts T, Surján G, Vicsek T, et al. A nationwide study of the epidemiology of relapsing polychondritis. Clin Epidemiol 2016;8:211-30.

2. Hazra N, Dregan A, Charlton J, Gulliford MC, D'Cruz DP. Incidence and mortality of relapsing polychondritis in the UK: a population-based cohort study. Rheumatology 2015;54:2181-7.

3. Mathew SD, Battafarano DF, Morris MJ. Relapsing polychondritis in the Department of Defense population and review of the literature. Semin Arthritis Rheum 2012;42:70-83.

4. de Montmollin N, Dusser D, Lorut C, Dion J, Costedoat-Chalumeau $\mathrm{N}$, Mouthon L, et al. Tracheobronchial involvement of relapsing polychondritis. Autoimmun Rev 2019;18:102353.

5. Rednic S, Damian L, Talarico R, Scirè CA, Tobias A, Costedoat-Chalumeau N, et al. Relapsing polychondritis: state of the art on clinical practice guidelines. RMD Open 2018;4 Suppl 1:e000788.

6. Le Besnerais M, Arnaud L, Boutémy J, Bienvenu B, Lévesque H, Amoura Z, et al. Aortic involvement in relapsing polychondritis. Joint Bone Spine 2018;85:345-51.

7. Tomelleri A, Campochiaro C, Sartorelli S, Papa M, De Luca G, Cavalli $\mathrm{G}$, et al. Large vessel vasculitis affecting the aorta and its branches in relapsing polychondritis: case series and systematic review of the literature. J Rheumatol 2020;47:1780-4.

8. Erdogan M, Esatoglu SN, Hatemi G, Hamuryudan V. Aortic involvement in relapsing polychondritis: case-based review. Rheumatol Int 2019 Nov 25 (E-pub ahead of print).

9. Sharma A, Kumar R, Mb A, Naidu GS, Sharma V, Sood A, et al. Fluorodeoxyglucose positron emission tomography/computed tomography in the diagnosis, assessment of disease activity and therapeutic response in relapsing polychondritis. Rheumatology 2020;59:99-106.

10. Dejaco C, Ramiro S, Duftner C, Besson FL, Bley TA, Blockmans D, et al. EULAR recommendations for the use of imaging in large vessel vasculitis in clinical practice. Ann Rheum Dis 2018; 77:636-43.

11. Jennette JC, Falk RJ, Bacon PA, Basu N, Cid MC, Ferrario F, et al. 2012 revised International Chapel Hill Consensus Conference Nomenclature of Vasculitides. Arthritis Rheum 2013;65:1-11. 